\title{
Discrepancy of Products of Hypergraphs
}

\author{
Benjamin Doerr ${ }^{1}$, Michael Gnewuch ${ }^{2 \dagger}$ and Nils Hebbinghaus ${ }^{3 \ddagger}$ \\ ${ }^{1}$ Max-Planck-Institut für Informatik, Stuhlsatzenhausweg 85, D-66123 Saarbrücken, e-mail: doerr@mpi-sb.mpg.de \\ ${ }^{2}$ Max-Planck-Institut für Mathematik in den Naturwissenschaften, Inselstraße 22, D-04103 Leipzig, e-mail: \\ gnewuch@mis.mpg.de \\ ${ }^{3}$ Institut für Informatik und Praktische Mathematik, Christian-Albrechts-Universität Kiel, Christian-Albrechts-Platz \\ 4, D-24118 Kiel,e-mail: nhe@numerik.uni-kiel.de
}

For a hypergraph $\mathcal{H}=(V, \mathcal{E})$, its $d$-fold symmetric product is $\Delta^{d} \mathcal{H}=\left(V^{d},\left\{E^{d} \mid E \in \mathcal{E}\right\}\right)$. We give several upper and lower bounds for the $c$-color discrepancy of such products. In particular, we show that the bound $\operatorname{disc}\left(\Delta^{d} \mathcal{H}, 2\right) \leq$ $\operatorname{disc}(\mathcal{H}, 2)$ proven for all $d$ in [B. Doerr, A. Srivastav, and P. Wehr, Discrepancy of Cartesian products of arithmetic progressions, Electron. J. Combin. 11(2004), Research Paper 5, 16 pp.] cannot be extended to more than $c=2$ colors. In fact, for any $c$ and $d$ such that $c$ does not divide $d$ !, there are hypergraphs having arbitrary large discrepancy and $\operatorname{disc}\left(\Delta^{d} \mathcal{H}, c\right)=\Omega_{d}\left(\operatorname{disc}(\mathcal{H}, c)^{d}\right)$. Apart from constant factors (depending on $c$ and $d$ ), in these cases the symmetric product behaves no better than the general direct product $\mathcal{H}^{d}$, which satisfies $\operatorname{disc}\left(\mathcal{H}^{d}, c\right)=O_{c, d}\left(\operatorname{disc}(\mathcal{H}, c)^{d}\right)$.

Keywords: discrepancy, hypergraphs, Ramsey theory

\section{Introduction}

We investigate the discrepancy of certain products of hypergraphs. In [2], Srivastav, Wehr and the first author noted the following. For a hypergraph $\mathcal{H}=(V, \mathcal{E})$ define the $d$-fold direct product and the $d$-fold symmetric product by

$$
\begin{aligned}
\mathcal{H}^{d} & :=\left(V^{d},\left\{E_{1} \times \cdots \times E_{d} \mid E_{i} \in \mathcal{E}\right\}\right), \\
\Delta^{d} \mathcal{H} & :=\left(V^{d},\left\{E^{d} \mid E \in \mathcal{E}\right\}\right) .
\end{aligned}
$$

Then for the (two-color) discrepancy

$$
\operatorname{disc}(\mathcal{H}):=\min _{\chi: V \rightarrow\{-1,1\}} \max _{E \in \mathcal{E}}\left|\sum_{v \in E} \chi(v)\right|,
$$

\footnotetext{
${ }^{\dagger}$ Supported by the Deutsche Forschungsgemeinschaft, Grant SR7/10-1.

$\ddagger$ Supported by the Deutsche Forschungsgemeinschaft, Graduiertenkolleg 357. 
we have

$$
\operatorname{disc}\left(\mathcal{H}^{d}\right) \leq \operatorname{disc}(\mathcal{H})^{d} \quad \text { and } \quad \operatorname{disc}\left(\Delta^{d} \mathcal{H}\right) \leq \operatorname{disc}(\mathcal{H})
$$

In this paper, we show that the situation is more complicated for discrepancies in more than two colors. In particular, it depends highly on the dimension $d$ and the number of colors, whether the discrepancy of symmetric products is more like the discrepancy of the original hypergraph or the $d$-th power thereof. Let us make this precise:

For $c \in \mathbb{N}_{\geq 2}$, a $c$-coloring of a hypergraph $\mathcal{H}=(V, \mathcal{E})$ is a mapping $\chi: V \rightarrow[c]$, where $[r]:=\{n \in$ $\mathbb{N} \mid n \leq r\}$ for any $r \in \mathbb{R}$. The discrepancy problem asks for balanced colorings of hypergraphs in the sense that each hyperedge shall contain the same number of vertices in each color. The discrepancy of $\chi$ and the $c$-color discrepancy of $\mathcal{H}$ are defined by

$$
\begin{aligned}
\operatorname{disc}(\mathcal{H}, \chi) & :=\max _{E \in \mathcal{E}} \max _{i \in[c]}|| \chi^{-1}(i) \cap E\left|-\frac{1}{c}\right| E||, \\
\operatorname{disc}(\mathcal{H}, c) & :=\min _{\chi: V \rightarrow[c]} \operatorname{disc}(\mathcal{H}, \chi) .
\end{aligned}
$$

These notions were introduced in [1] extending the discrepancy problem for hypergraphs to arbitrary numbers of colors. Note that $\operatorname{disc}(\mathcal{H})=2 \operatorname{disc}(\mathcal{H}, 2)$ holds for all $\mathcal{H}$. In this more general setting, the product bound proven in [2] is

$$
\operatorname{disc}\left(\mathcal{H}^{d}, c\right) \leq c^{d-1} \operatorname{disc}(\mathcal{H}, c)^{d} .
$$

However, as we show in this paper the relation $\operatorname{disc}\left(\Delta^{d} \mathcal{H}, c\right)=O(\operatorname{disc}(\mathcal{H}, c))$ does not hold in general. We give a characterization of those values of $c$ and $d$, for which it is fulfilled for every hypergraph $\mathcal{H}$. In particular, we present for all $c, d, k$ such that $c$ does not divide $d$ ! a hypergraph $\mathcal{H}$ having $\operatorname{disc}(\mathcal{H}, c) \geq k$ and $\operatorname{disc}\left(\Delta^{d} \mathcal{H}, c\right)=\Omega_{d}\left(k^{d}\right)$. In the light of (1), this is largest possible apart from factors depending on $c$ and $d$ only.

On the other hand, there are further situations where this worst case does not occur. We state some results of this type in the last section.

\section{Symmetric Direct Products Having Large Discrepancy}

Let $S(d, l), d, l \in \mathbb{N}$, denote the Stirling numbers of the second kind. For $c \in \mathbb{N}$ and $\lambda \in N_{0}$ we write $c \mid \lambda$ if there exists an $m \in \mathbb{N}_{0}$ with $m c=\lambda$.

Theorem 1 Let $c, d \in \mathbb{N}$.

If $c \mid k ! S(d, k)$ for all $k \in\{2, \ldots, d\}$, then every hypergraph $\mathcal{H}$ satisfies

$$
\operatorname{disc}\left(\Delta^{d} \mathcal{H}, c\right) \leq \operatorname{disc}(\mathcal{H}, c) .
$$

If $c \nmid k ! S(d, k)$ for some $k \in\{2, \ldots, d\}$, then there exists a hypergraph $\mathcal{K}$ such that

$$
\operatorname{disc}\left(\Delta^{d} \mathcal{K}, c\right) \geq \frac{1}{3 k !} \operatorname{disc}(\mathcal{K}, c)^{k},
$$

and $\mathcal{K}$ can be chosen to have arbitrary large discrepancy $\operatorname{disc}(\mathcal{K}, c)$. 
We state some simple consequences of Theorem 1:

Corollary 2 (a) Let $d \geq 3$ be an odd number. Then $\operatorname{disc}\left(\Delta^{d} \mathcal{H}, 3\right) \leq \operatorname{disc}(\mathcal{H}, 3)$ holds for any hypergraph $\mathcal{H}$.

(b) Let $d \geq 2$ be an even number and $c=3 l, l \in \mathbb{N}$. There exists a hypergraph $\mathcal{H}$ with arbitrary large discrepancy that fulfills $\operatorname{disc}\left(\Delta^{d} \mathcal{H}, c\right) \geq \frac{1}{6} \operatorname{disc}(\mathcal{H}, c)^{2}$.

Proof: Obviously $3 \mid k$ ! for all $k \geq 3$. Since $S(d, 2)=2^{d-1}-1$, we have $3 \mid S(d, 2)$ if and only if $d$ is odd.

Corollary 3 Let $l \in \mathbb{N}$ and $c=4 l$. For all $d \geq 2$ there exists a hypergraph $\mathcal{H}$ with arbitrary large discrepancy such that $\operatorname{disc}\left(\Delta^{d} \mathcal{H}, c\right) \geq \frac{1}{6} \operatorname{disc}(\mathcal{H}, c)^{2}$.

Proof: As $S(d, 2)=2^{d-1}-1$ is an odd number, we have $4 \nmid 2 ! S(d, 2)$.

Our proof of Theorem 1 uses the following lemma.

Lemma 4 Let $c, d \in \mathbb{N}$. For all $m \in \mathbb{N}$ there exists an $n \in \mathbb{N}$ having the following property: For each $c$ coloring $\chi:[n]^{d} \rightarrow[c]$ we find a subset $T \subseteq[n]$ with $|T|=m$ such that for all $l \in[d]$ each l-dimensional simplex in $T^{d}$ is monochromatic with respect to $\chi$.

Hereby an $l$-dimensional simplex in $T^{d}$ is of the following form: Fix a partition $\left\{J_{1}, \ldots, J_{l}\right\}$ of $[d]$ and define vectors $f^{(i)}$ by $f_{k}^{(i)}=1$ if $k \in J_{i}$ and 0 else. Then

$$
S:=\left\{\sum_{i=1}^{l} \alpha_{i} f^{(i)} \mid \alpha_{1}, \ldots, \alpha_{l} \in T, \alpha_{1}<\ldots<\alpha_{l}\right\}
$$

is an $l$-dimensional simplex in $T^{d}$. The proof of Lemma 4 is based on an argument from Ramsey theory (see, e.g., [3, Section 1.2]).

Related to Lemma 4 is a result of Gravier, Maffray, Renault and Trotignon [4]. They have shown that for any $m \in \mathbb{N}$ there is an $n \in \mathbb{N}$ such that any collection of $n$ different sets contains an induced subsystem on $m$ points such that one of the following holds: (a) each vertex forms a singleton, (b) for each vertex there is a set containing all $m$ points except this one, or (c) by sufficiently ordering the points $p_{1}, \ldots, p_{m}$ we have that all sets $\left\{p_{1}, \ldots, p_{\ell}\right\}, \ell \in[m]$, are contained in the system..$^{\S}$

In our language, this means that any 0,1 matrix having $n$ distinct rows contains a $m \times m$ submatrix that can be transformed through row and column permutations into a matrix that is (a) a diagonal matrix, (b) the inverse of a diagonal matrix, or (c) a triangular matrix.

$\S$ To be precise, the authors also have the empty set contained in cases (a) and (c) and the whole set in case (b). It is obvious that by altering $m$ by one, one can transform one result into the other. 
Hence this result is very close to the assertion of Lemma 4 for dimension $d=2$ and $c=2$ colors. It is stronger in the sense that not only monochromatic simplices are guaranteed, but also a restriction to 3 of the 8 possible color combinations for the 3 simplices is given. Of course, this stems from the facts that (a) column and row permutations are allowed, (b) not a submatrix with index set $T^{2}$ is provided but only one of type $S \times T$, and (c) the assumption of having different sets ensures sufficiently many entries in both colors.

\section{Further Upper Bounds}

Besides the first part of Theorem 1, there are more ways to obtain upper bounds.

Theorem 5 Let $\mathcal{H}=(V, \mathcal{E})$ be a hypergraph. Let $p$ be a prime number, $q \in \mathbb{N}$ and $c=p^{q}$. Furthermore, let $d \geq c$ and $s=d-(p-1) p^{q-1}$. Then $\operatorname{disc}\left(\Delta^{d} \mathcal{H}, c\right) \leq \operatorname{disc}\left(\Delta^{s} \mathcal{H}, c\right)$.

As a corollary, we state a less general (but also less technical) version of Theorem 5:

Corollary 6 Let $\mathcal{H}=(V, \mathcal{E})$ be a hypergraph. If $c$ is a prime number, $q \in \mathbb{N}$ and $d=c^{q}$, then $\operatorname{disc}\left(\Delta^{d} \mathcal{H}, c\right) \leq \operatorname{disc}(\mathcal{H}, c)$.

Proof: Use $c^{q}=1+(c-1) \sum_{j=0}^{q-1} c^{j}$ and Theorem 5 (repeatedly).

The following result is an extension of the first statement of Theorem 1.

Theorem 7 Let $c, d \in \mathbb{N}$, and let $d^{\prime} \in\{2 \ldots, d\}$. If $c \mid k ! S\left(d^{\prime}, k\right)$ for all $k \in\left\{2, \ldots, d^{\prime}\right\}$, then

$$
\operatorname{disc}\left(\Delta^{d} \mathcal{H}, c\right) \leq \operatorname{disc}\left(\Delta^{d-d^{\prime}+1} \mathcal{H}, c\right)
$$

holds for every hypergraph $\mathcal{H}$.

Remark 8 The condition in Theorem 7 is only sufficient but not necessary for the validity of (4), as the following example shows: Let $c=4, d \geq c$ and $d^{\prime}=3$. According to Theorem 5, we get for each hypergraph $\mathcal{H}$ that $\operatorname{disc}\left(\Delta^{d} \mathcal{H}, c\right) \leq \operatorname{disc}\left(\Delta^{d-2} \mathcal{H}, c\right)=\operatorname{disc}\left(\Delta^{d-d^{\prime}+1} \mathcal{H}, c\right)$. But we have $2 ! S\left(d^{\prime}, 2\right)=$ $6=3 ! S\left(d^{\prime}, 3\right)$ and $4 \nmid 6$. This example indicates also that the proof methods of Theorem 5 and Theorem 7 are different.

\section{References}

[1] B. Doerr and A. Srivastav, Multi-Color Discrepancies, Comb. Probab. Comput. 12(2003), 365399.

[2] B. Doerr, A. Srivastav, and P. Wehr, Discrepancy of Cartesian products of arithmetic progressions, Electron. J. Combin. 11(2004), Research Paper 5, 16 pp. 
[3] R. L. Graham, B. L. Rothschild, and J. H. Spencer, Ramsey Theory, Second Edition, Wiley, New York, USA, 1990.

[4] S. Gravier, F. Maffray, J. Renault, and N. Trotignon, Ramsey-type results on singletons, cosingletons and monotone sequences in large collections of sets, European J. Combin. 25 (2004), 719-734. 
